\title{
Using Subtitles to Enliven Reading
}

\author{
Yanling Hwang (Corresponding author) \\ School of Applied Foreign Languages, Chung Shan Medical University \\ 100, Sec. 2, Dah-Ching St., Taichung, Taiwan \\ Tel: 886-4-2473-20088 ext. 17132 E-mail: yanling_h@yahoo.com \\ Peiwen Huang \\ Department of Applied Foreign Languages, National Formosa University \\ No.64, Wunhua Rd., Huwei Township, Yunlin County 632, Taiwan \\ Tel: 886-5-631-5000Ｅ-mail: phuang@nfu.edu.tw
}

Received: March 3, 2011 Accepted: March 24, 2011 doi:10.5539/ells.v1n1p2

\begin{abstract}
There are an increasing number of foreign language teaching techniques that integrate with the latest technology, such as computers, video materials. As the emphasis in multimedia shifts to success for all language learners, educators tend to carry out various techniques to demonstrate benefits. Presenting subtitles aids visual channels to communicate verbal information. The presenter examines whether video English captions improve or impede EFL students' reading comprehension.

Using the instructional videos with English subtitles 1 hour every 2 weeks over 10 weeks. Two versions of videos, one with captioning and one without it, were used by two groups randomly selected among freshmen at the university in Taiwan. Reading comprehension test- General English Proficiency Test (GEPT) - intermediate- was administered by participants in order to determine the influences of using subtitles on learners' reading comprehension.
\end{abstract}

Keywords: Subtitles, English learning, Reading comprehension

\section{Introduction}

There are an increasing number of foreign language teaching techniques that integrate with the latest technology, such as computers, video materials. In other words, the movement of language teaching goals and practices has shifted from the printed word and knowledge of language systems to the use and communicative value of the spoken language in the everyday setting (Vanderplank, 1993). In this particular setting, learners gradually develop their language acquisition by being exposed to the genuine environment of the target language. Many researchers have claimed that multimedia have beneficial effects on language learning because of abundant and authentic comprehensible input.

Likewise, television programs and videotapes have found a place in the transmission of information and are powerful tools in promoting language teaching (Anderson \& Lorch, 1983). The subtitles or captions, a key function on television and videotapes, are synchronized with the dialogue or narration of the program's audio track, augmenting and understanding of TV programs and videos. The terms subtitles and captions are interchangeable in this paper and are defined as the translations of the spoken word to the written with the identical language shown at the bottom of the television screen. Lambert, Boehler and Sidoti (1981) have asserted that the stable general trend indicates that information coming through two input form is more thoroughly processed than if either dialogue or captions are presented alone. Moreover, Paivio (1971) proposed the dual-coding theory which suggests that when pictures are added to the message the number of cues associated with the message increases. Viewers then will be more likely to remember the message. In that sense the results of past research seem to sustain the aspect that the use of captions triggers multi-sensory processing, interacting with audio, video and print components.

Despite much research has hypothesized the benefits from the use of captions for language learners, little research related to this interest has found in Taiwan. Thus, there is a need for exploration. In addition, as the emphasis in multimedia shifts to success for all language learners, educators tend to carry out various techniques to demonstrate benefits. Presenting subtitles aids visual channels to communicate verbal information. The present study investigated whether video English captions improve or impede EFL students' reading comprehension. Using the 
instructional videos with English subtitles 1 hour every 2 weeks over 10 weeks. Two versions of videos, one with captioning and one without it, were used by two groups randomly selected among freshmen at the university in Taiwan. Reading comprehension test - General English Proficiency Test (GEPT) - intermediate was administered in order to determine the influences of using subtitles on learners' reading comprehension.

\section{Literature Reviews}

\subsection{Dual-coding Theory}

Allan Paivio (1971) proposed the dual-coding theory (DCT) as an attempt to explain how learners use associations when the pictorial and linguistic information are processed differently. Inclined toward giving equal weight to verbal and non-verbal processing, Paivio asserted that information could be represented by pictures and words at the same time. The two information sources trigger two coding systems: visual codes and verbal codes which are functionally independent and interconnected by referential links (see Figure 1). The theory assumes that there are two cognitive subsystems: one subsystem is specialized for verbal information and the other is specialized for non-verbal information.

This concept of independent verbal and imagery representation systems is at the heart of Paivio's dual-coding model. According to this model, Paivio postulated the two different recreational units: imagens for mental images referring to any concrete stimuli and logogens for verbal entities containing abstract stimuli that include vague visual as well as verbal information. Paivio contended that imagens are coded twice, both as images and corresponding verbal labels, whereas logogens are more difficult to image and are coded only as verbal information. DCT claims that imagens are faster and easier to recall than the logogen which is structured in discrete, sequential units. In other words, dual-coding theory evolved from Paivio's studies on the role of imagery in associate learning, supporting the learning effectiveness of illustrated texts and proposing that information is much easier to retain and retrieve when dual-coded systems occurs. The result indicates that the availability of two mental representations instead of one promotes comprehension

In this regard, the use of captioned videos demonstrating pictures and words in an auditory form and in a visual form simultaneously enhances the comprehension for viewers. Moreover, if pictorial cues are redundant with the audio message, then viewers will be more likely to remember the message.

\subsection{ACT Model}

Based on a cognitive view of language development, language acquisition is regarded as a gradual automitization of skills through stages of restructuring and linking new information to old knowledge. John Anderson (1983) describes the process of learning development from declarative to procedural knowledge. He distinguishes between two different kinds of knowledge, being necessary for second language learning. Declarative knowledge refers to knowledge that is conscious and stored as a series of statements or images. It is knowledge about rules, words and facts, whereas procedural knowledge refers to knowledge that is unconscious and consists of routines or procedures that allow us to bring declarative knowledge into use. For example, our procedural knowledge of our mother tongue permits us to construct grammatically correct sentences without consciously thinking about it.

Moreover, Anderson also points out that learning takes place in three stages. First, the cognitive stage describes that the learner needs to explicitly utilize mental processes to access knowledge. In the case of the language example, that an $-\mathrm{s}$ must be added to a verb after a third person subject. In this stage, the acquisition process is likely to involve many errors, and hesitant fluency. Second, the associative stage asserts that the learner can more readily correct their errors, and produce language. There is a more comprehensive source of knowledge to access in comprehending and producing language. For example, how to add $-\mathrm{s}$ when the context requires it. Last, the autonomous stage claims that the performance becomes native-like, with errors free. The mental processes needed to access knowledge have become implicit. Learners might not be able to explain what they are doing.

Briefly stated, the ACT model views second language learning as a movement from declarative to procedural knowledge, which takes place in three stages stated above. The three stages can be characterized to be from conscious, slow and incorrect to unconscious, fast and correct.

In short, Anderson claims that language learning involves a shift from the cognitive stage toward the autonomous state processing via practice, and initial practice during the cognitive and associative stages lies in proceduralization and initial automation of a new skill.

\subsection{Research on L2 captions on reading comprehension}

In Rochester, NY, Parlato (1985) used in-class captioned TV viewing as a group activity that provided a common 
frame of reference or talking point from which to build vocabulary and concepts. Subjects viewed programs, looked for differences between captions and dialogue, and discussed these differences after the viewing. This activity helped learners improve their reading fluency and metalinguistic knowledge about how language can be used and manipulated. In accordance with the above mentioned finding, Goldman (1993) contended that CCTV motivated ESL students of intermediate and advanced levels in terms of their reading comprehension. In addition, CCTV is a powerful and dynamic supplemental teaching aid (Goldman, 1996).

\subsection{Research on L1 captions on reading comprehension}

In addressing the effectiveness of the presence of captions, d'Ydewalle, Praet, Verfaillie, and Van Rensbergen (1991) conducted a study pertaining to the relationship between subtitled televisions and reading behavior. American and Dutch-speaking subjects who differ in terms of subtitling familiarity participated in this study. In experiment 1 , American subjects watched an American movie with English subtitles. The authors asserted that Americans spend considerable time in the subtitled area in spite of their lack of familiarity with subtitles. In experiment 2, a movie in Dutch with Dutch subtitles was viewed by Dutch-speaking subjects. As a result, they suggested that reading subtitles is preferred because of efficiency in following and understanding the movie.

Similarly, to investigate the effects of various translation methods used in imported instructional video programs on Taiwan elementary school students' visual and verbal memory, Tyan (1995) assigned 128 fourth grade students into 5 different modes of presentation. The five groups were (i) Chinese narration, with no subtitles, (ii) English narration, with complete Chinese subtitles, (iii) Chinese narration, with complete Chinese subtitles, (iv) Chinese narration, with key-words subtitled in Chinese, and (v) English narration, with no subtitles. The results of this study revealed that groups viewing Chinese narration with no subtitles significantly outperformed the English narration group on the posttest.

\section{Methodology}

\subsection{Participants}

The participants of this study were 80 freshman students from a college in central Taiwan whose average age was 19. They are male and female students in two groups. The minimum of length of learning English is about seven years. All of the participants enrolled the English comprehension class as one of the required courses designed for freshman students once a week. The participants were randomly assigned to one of the two different condition groups. Each group consisted of 40 students.

\subsection{Instruments}

Two instruments were administered in this study and were introduced in detail as follows. The first instrument was the Content-Specific Test (CST) for assessing the language comprehension on a particular video episode. The construct of the CST developed by the researcher focused on the student's comprehension of the major concepts in the fifth video episode, especially on listening comprehension, vocabulary recognition and content comprehension. The second instrument utilized in the study was the practice sample of the General English Proficiency Test (GEPT) - intermediate for measuring students' overall language proficiency. The GEPT is a standardized test established by the Ministry of Education in Taiwan to evaluate the student's overall language proficiency in terms of listening and reading skills.

\subsection{Procedures}

Firstly, the researcher planned the first meeting with the cooperating teacher, discussing who the participants were and what instructions should be used in the class. Secondly, teacher was asked to send syllabi to the research for both classes in order to ensure consistency of instructions in both groups. Thirdly, the cooperating teacher randomly divided participants in two groups. One group, an experimental group, watched the English videos with captions; whereas, the control group watched it without captions. Prior to the date of the actual experiment, participants in the two groups announced they would take part in the study and explained the goal and the purpose of the study. They were asked to take the practice sample of the GEPT - intermediate. After the fifth episode was viewed in class, participants in both groups were given the CST immediately. Finally, during the fifth week, the practice sample of the GEPT- intermediate, one identical to a pretest, was administered again to the control group and the experimental group. The SPSS statistical package was used to analyze the data in this study after the test data were received. An analysis of covariance (ANCOVA) statistical method was utilized in the study to control for extraneous variables and any bias which might be attributed to the randomization process. The results of ANCOVA showed the difference between two groups. The level of significance was set at 0.05 . 


\section{Data Findings}

\subsection{Subtest of the GEPT- Reading Comprehension Results}

The raw scores on multiple-choice test items in the reading comprehension subtest were analyzed for a possible high score of 15. Table 1 presented the means and the standard deviations of the two groups on GEPT. The description reported that the experimental group outperformed the comparison group $(\mathrm{M}=6.80 ; \mathrm{SD}=2.41 \mathrm{vs} . \mathrm{M}=5.70 ; \mathrm{SD}=$ 2.69 , respectively).

Applying the same statistical procedures on the raw scores of the composite GEPT and GEPT listening subtest, an analysis of covariance (ANCOVA) was done to determine whether the two conditions differed significantly from each other on the scores of the reading subtest. The results of this statistical analysis were presented in Table 2. As a result, an ANCOVA showed no significant differences between the mean reading scores of the experimental and that of control group ( $\mathrm{df}=1, \mathrm{~F}=3.24, \mathrm{p}=.076)$. Therefore, the null hypothesis was retained.

\section{Discussion}

Compared to the mean reading GEPT scores of the experimental and the control group, the results revealed no significant disparity between participants' reading performances on both conditions after the experiment. The findings address the fact that students watching captioned videos did not score higher on the GEPT - reading subtest than those who watched non-captioned videos over a period of five weeks. This conclusion led to the acceptance of the null hypothesis. According to this finding, the result of this hypothesis differed from that obtained by Goldman (1993), examining the relationship between ESL students of intermediate and advanced levels' reading comprehension. Goldman showed that the CCTV improved students' reading performance.

\section{Conclusion}

This study aimed to investigate whether video English captions improve or impede EFL students' reading comprehension. Possible reasons for a failure to reject null hypothesis are two-fold. One possibility might be that the single channel learning is inferior to multiple-channel learning. On the practice sample GEPT - intermediate reading subtest - text is processed through only one channel. One representation is not thought to be as comprehensible as two representations. Thus, information encoded is not easy to associate and retrieve. According to Hartman (1961), print alone might be beneficial for literate students. The subjects in this study were still in a language learning processing phrase and were far from being masters of the target language.

Secondly, the length of practice on the captioning viewing was too short to draw significant conclusions about the reading performance in the experimental group. In the ACT model, Anderson (1983) asserts that initial practice during the cognitive and associative stages lies in proceduralization and initial automation of a new skill. Therefore, it is suggested that caption viewing, regarded as one learning strategy, is meant to be introduced and practiced as often as possible in the experimental group for the automation of the new skill of watching videos with captions. Even though learners are exposed to authentic and contextually rich materials, they still receive insufficient input from authentic exposure. Therefore, even if input is considered comprehensible, it is not necessarily accessible for effective reading proficiency. While the results in this study indicate that captioned videos did not affect students' reading proficiency after five weeks, it was hard to establish the cause and effect relationship between the presentation of captioned videos and the experimental group's reading abilities. To ensure efficient and effective captioned materials for learners' reading performance, exposure to an authentic environment and a great deal of practice on caption viewing should be achieved and remain consistent. This leads to significant reading improvement.

In short, captioned materials as a medium for teaching reading skills are limited to having theoretical justification as well as practical application.

\section{References}

Anderson, D., \& Lorch, E. (1983). Looking at television action or relation. In Bryant \& D. Anderson (Eds.), Children's understanding of television: Research on attention and comprehension (pp. 1-33). New York: Academic Press.

Anderson, J. (1983). The architecture of cognition. Cambridge, MA: Harvard University Press.

Lambert, W., Boehler, I., \& Sidoti, N. (1981). Choosing the languages of subtitles and spoken dialogues for media presentations: Implication for second langue education. Applied Psycholinguistics, 2, 133-148.

Goldman, M. (1993). Using captioned TV for teaching reading. Fastback, 359. Phi Delta Kappa Educational Foundation.

Goldman, M. (1996). If you can read this, thank TV. TESOL Journal, 6(2), 15-18. 
Hartman, F. (1961). Single and multiple channel communication: A review of research and a proposed model. Audio Visual Communications Review, 9(6), 235-262.

Paivio, A. (1971). Imagery and cognitive process. New York: Holt, Rinehart \& Winston.

Parlato, S. (1985). Re-discovering films with captions. Teaching English to Deaf and Second-Language Students, $3(1), 17-20$.

Vanderplank, R. (1993). A very verbal medium: Language learning through closed captions. TESOL Journal, 3(1), $10-14$.

Table 1.Mean Reading Scores on GEPT

\begin{tabular}{|l|l|l|l|}
\hline Group & $\mathrm{N}$ & $\mathrm{M}$ & $\mathrm{SD}$ \\
\hline Caption & 40 & 6.80 & 2.41 \\
\hline Non-caption & 40 & 5.70 & 2.69 \\
\hline
\end{tabular}

Table 2. ANCOVA: Scores on the Reading Subtest of the GEPT

\begin{tabular}{|l|l|l|l|l|l|}
\hline Source & $\begin{array}{l}\text { Sum of } \\
\text { Squares }\end{array}$ & Df & $\begin{array}{l}\text { Mean } \\
\text { Squares }\end{array}$ & F-Value & P-Value \\
\hline Pretest & 46.98 & 1 & 46.98 & 7.80 & .006 \\
\hline Group & 19.43 & 1 & 19.43 & 3.24 & .076 \\
\hline Error & 461.83 & 77 & 5.99 & & \\
\hline
\end{tabular}

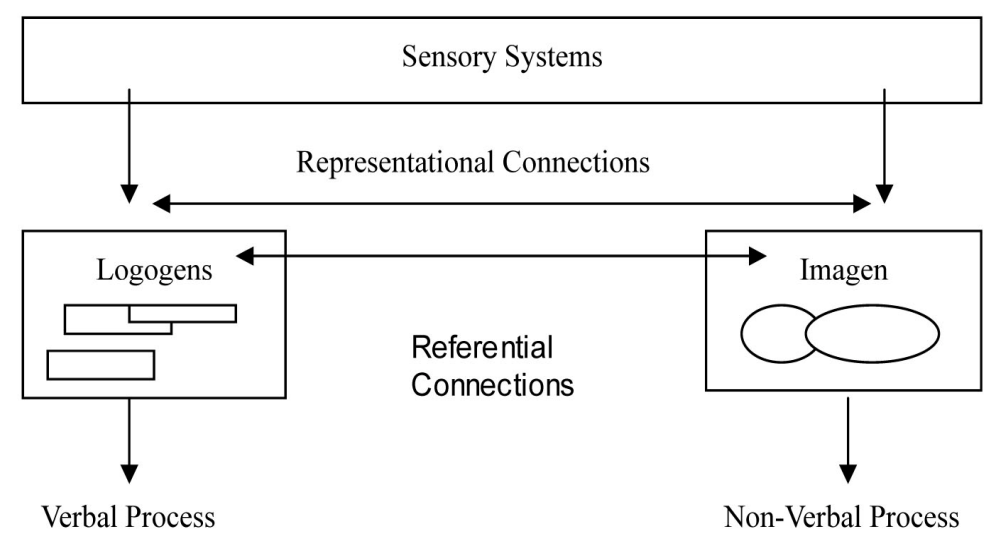

Figure 1. Paivio's Dual-Coding Theory 